\title{
The HoSTS Project: A Homogeneous Study of Transiting Systems
}

\author{
Yilen Gómez Maqueo Chew ${ }^{1,2}$, Francesca Faedi' ${ }^{2}$, Leslie Hebb ${ }^{3}$, \\ Don Pollacco ${ }^{2}$, Keivan Stassun ${ }^{1}$, Phillip Cargile ${ }^{1}$, Barry Smalley ${ }^{4}$, \\ Amanda Doyle ${ }^{4}$, Luan Ghezzi ${ }^{5}$, Susana C. C. Barros ${ }^{6}$, and \\ Andrew Collier Cameron ${ }^{7}$ \\ ${ }^{1}$ Department of Physics \& Astronomy, Vanderbilt University, Nashville, TN 37235, USA \\ ${ }^{2}$ Department of Physics, University of Warwick, Coventry CV4 7AL, UK \\ email: y.gomez@warwick.ac.uk \\ ${ }^{3}$ Department of Astronomy, University of Washington, Seattle, WA 98195, USA \\ ${ }^{4}$ Astrophysics Group, Keele University, Staffordshire ST5 5BG, UK \\ ${ }^{5}$ Observatório Nacional, Rua Gal. José Cristino 77, Rio de Janeiro, RJ 20921-400, Brazil \\ ${ }^{6}$ Laboratoire d'Astrophysique de Marseille UMR 7326, 13388, Marseille, France \\ ${ }^{7}$ School of Physics and Astronomy, University of St Andrews, St Andrews, Fife KY16 9SS, UK

\begin{abstract}
The Homogeneous Study of Transiting Systems (HoSTS) will derive a consistent and homogeneous set of both the stellar and planetary physical properties for a large sample of bright transiting planetary systems with confirmed planetary masses and measured radii. Our resulting catalogs of the fundamental properties of these bright planets and their host stars will enable us to explore empirical correlations that will lead to a better understanding of planetary formation and evolution. We present our pilot study of the planet-hosting star WASP-13, and the framework of our project which will allow for the identification of true relationships among the physical properties of the systems from any systematics.
\end{abstract}

Keywords. stars: fundamental parameters, planetary systems, techniques: radial velocities techniques: spectroscopic, techniques: techniques: photometric, stars: individual (WASP-13)

\section{Introduction}

Over 300 transiting extrasolar planets have been discovered thus far, exhibiting an amazing array of physical properties orbiting stellar hosts that also span a range of properties and stages of evolution. This diversity of planets and of the stellar environments in which they form and evolve offers a watershed opportunity to develop a systematic understanding of the physics that governs planet formation, structure, and evolution, and of the connections between the planets and their host stars. To fully exploit the wealth of information encoded in the set of transiting planetary systems requires careful determination of precise fundamental properties of the exoplanets and their host stars.

We have underway a systematic investigation to fully and accurately characterize a large sample of the known bright transiting planetary systems. These transiting systems, in which part of the stellar light is blocked as a planet crosses the disk of the star, are extremely valuable for detailed study because they are the only systems that allow for precise measurements of the mass and radius of the planet. For the first time, there is now a large enough sample of transiting extra-solar planets for which both mass and radius measurements can be accurately determined so that statistically significant studies of their physical properties can be achieved. Thus, we present the homogeneous methodology that will be employed in our project, exemplified by the results from the pilot study of the transiting system WASP-13 (Gómez Maqueo Chew et al., 2013) which highlights 
the importance of the proposed project in order to identify, and tighten empirical relationships among the physical properties of the exoplanet systems. HoSTS will cover the parameter space of known physical properties of planetary systems for the stellar hosts (e.g., $T_{\text {eff }},[\mathrm{Fe} / \mathrm{H}]$, evolutionary status, and in single and multiple stellar systems), with single and multiple planets exhibiting a range of masses and radii. Our project extends previous efforts (e.g., Torres et al. (2012)) in that it will derive consistently both stellar and planetary properties from high quality data.

\section{Dataset and Methodology}

We have an extensive data set both obtained by our team, and compiled from the literature, for planetary systems in both the Northern and Southern hemispheres including: high resolution $(R>40 \mathrm{k})$, high signal-to-noise ratio $(\mathrm{S} / \mathrm{N}>200)$ echelle spectra with $\lambda \sim 4000-7800 \AA$, precise radial velocity measurements $(\sim 20 \mathrm{~m} / \mathrm{s})$, high cadence, high precision transit light curves, and an $\mathrm{H} \alpha$ long-slit spectra $(R>7 \mathrm{k}, \mathrm{S} / \mathrm{N}>200)$.

The methodology is described in detail in Gómez Maqueo Chew et al. (2013), and only briefly summarized here. The stellar effective temperature $\left(T_{\text {eff }}\right)$, the metallicity $([\mathrm{Fe} / \mathrm{H}])$, and the spectroscopic $\log g$ are derived from the high quality echelle spectrum using several stellar characterization methods based on the two techniques used in the exoplanet literature, spectral synthesys and the ionization/excitation equilibrium of iron lines. The stellar temperature will be compared against the value derived from the $\mathrm{H} \alpha$ spectrum as a check on $T_{\text {eff }}$. Using the stellar density ( $\rho_{\star}$; Seager \& Mallén-Ornelas, 2003) directly measured from the transit light curve, and the spectroscopically-determined $T_{\text {eff }}$ and $[\mathrm{Fe} / \mathrm{H}]$, the stellar mass is derived from stellar models (e.g., Dotter et al., 2008), and/or empirical relationships (e.g., Enoch et al., 2010). The planet properties are derived from the simultaneous modeling of the radial velocity and transit light curves using a MonteCarlo Markov Chain algorithm (Collier Cameron et al., 2007). Furthermore, HoSTS will explore the dependence of the derived physical properties on the dataset, on the stellar characterization method and the assumptions used (e.g., constraining spectroscopic log $g$ with $\rho_{\star}$ like suggested by Torres et al. (2012) for synthesis-based methods), and/or on the kind of planetary system.

\section{Conclusions from Pilot Study of WASP-13}

Our detailed study of WASP-13 showcases the importance of the HoSTS project for the tightening of the empirical correlations (see Fig. 4 in Gómez Maqueo Chew et al., 2013). Although our measurements of the planetary radius of WASP-13b and the metallicity of its host overlap with previous estimates, the errors on $[\mathrm{Fe} / \mathrm{H}]$ are significantly improved. Similar improvements for the larger sample of the transiting planets will allow the assessment of the validity of the observed trends, and the identification of any systematics. Moreover, we have included realistic uncertainties in the stellar mass, and consequently the planetary properties, including the contribution from the uncertainty in $[\mathrm{Fe} / \mathrm{H}]$, and in the stellar evolutionary status. Both of these sources of uncertainties are typically not taken into account. In the case of WASP-13, these uncertainties are shown to be the limiting factor in the precision that can be obtained for the planet properties.

\section{References}

Collier Cameron, A., et al. 2007, MNRAS, 380, 1230

Dotter, A., Chaboyer, B., Jevremović, D., et al., 2008, ApJS, 178, 89

Enoch, B., Collier Cameron, A., Parley, N. R., \& Hebb, L. 2010, A $\mathscr{G} A$, 516, A33

Gómez Maqueo Chew, Y., Faedi, F., Cargile, P., et al., 2013, ApJ, 768, 79

Seager, S. \& Mallén-Ornelas, G. 2003, ApJ, 585, 1038

Torres, G., Fischer, D. A., Sozzetti, A., et al. 2012, ApJ, 757, 161 